\title{
Íleo biliar resuelto por laparoscopia
}

\author{
Gallstone ileus resolved by laparoscopic approach \\ Daniel Ríos-Cruz, ${ }^{*}$ Fidel Alfonso Hernández-Linares, ${ }^{*}$ Natividad Cabrera-Valladares, ${ }^{\ddagger}$ \\ Sofía Magaly Flores-Hidalgo, ${ }^{\ddagger}$ Wendy López-Pérez, ${ }^{\ddagger}$ Myrtha Guadalupe Vera-Ruíz ${ }^{\ddagger}$
}

\author{
Palabras clave: \\ Íleo biliar, \\ laparoscopia, \\ colelitiasis. \\ Keywords: \\ Gallstone ileus, \\ laparoscopy, \\ cholelithiasis.
}

\footnotetext{
${ }^{*}$ Departamento de Cirugía, Hospital General Regional No. 1, Instituto Mexicano del Seguro Social. Cuernavaca, Morelos. ¥ Escuela de

Medicina, Universidad Latinoamericana.
} Cuernavaca, Morelos.

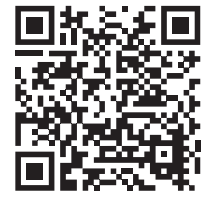

\section{RESUMEN}

Introducción: El íleo biliar es una obstrucción mecánica del tracto gastrointestinal, es causada por la impactación de uno o más litos de la vesícula biliar dentro de la luz intestinal a través de una fístula bilioentérica. Caso clínico: Mujer de 59 años con múltiples comorbilidades. Acude a urgencias por cuadro de obstrucción intestinal, mediante imagen se identifica neumobilia y distensión de asas intestinales, por lo que se decidió ingresar a quirófano por un probable íleo biliar y realizar laparoscopia diagnóstica, tras lo cual se encontró un lito de $3.5 \mathrm{~cm}$ de diámetro enclavado en íleon terminal. Conclusión: El íleo biliar es una complicación rara de la colelitiasis. El tratamiento integra la rehidratación y cirugía para corregir la causa de la obstrucción intestinal

\section{ABSTRACT}

Introduction: Gallstone ileus is a mechanical obstruction of the gastrointestinal tract caused by the impaction of one or more gallbladder stones inside the intestinal lumen through a bilioenteric-fistula. Clinical case: A 59-yearold woman with multiple comorbidities, who went to the emergency room for intestinal obstruction, pneumobileo and bowel distention are identified by image, so it was decided to enter the operating room for a probable biliary ileus and perform diagnostic laparoscopy, finding a $3.5 \mathrm{~cm}$ diameter lithium located in the terminal ileum. Conclusion: Gallstone ileus is a rare complication of cholelithiasis. The treatment integrates rehydration and surgical approach to correct the cause of intestinal obstruction.

\section{INTRODUCCIÓN}

E I ́́leo biliar (IB) se define como una obstrucción intestinal mecánica secundaria a la presencia de un cálculo biliar en la luz intestinal. ${ }^{1}$ Es una complicación rara y potencialmente grave de la colelitiasis, representa entre 1-4\% del total de las obstrucciones intestinales en el adulto. La causa más frecuente es la impactación del lito en el íleon tras su paso a través de una fístula bilioentérica, generalmente colecistoduodenal (68-95\%). ${ }^{2}$ Ésta aparece cuando hay episodios recurrentes de colecistitis aguda que generan inflamación y adhesiones entre la vesícula biliar y el tracto digestivo. ${ }^{3}$ En la mayoría de los casos, el cuadro obstructivo se produce en el íleon terminal (60\%), seguido del íleon proximal (25\%) y, de forma más rara, en el yeyuno (9\%), el colon sigmoide $(4 \%)$ o el duodeno $(2 \%) ;{ }^{4}$ está asociado con pacientes mayores de 65 años donde puede llegar a $25 \%$ de las obstrucciones de intestino delgado; ${ }^{5}$ es más prevalente en mujeres, con una proporción mujer a hombre de 3.6:1. La tasa de mortalidad asociada al íleo biliar abarca de 12 a 27\%, y la tasa de morbilidad alcanza $50 \%$, debido a la edad avanzada de los pacientes, a las patologías asociadas (usualmente severas), al ingreso hospitalario tardío y al tratamiento terapéutico retrasado. ${ }^{6}$

\section{CASO CLÍNICO}

Paciente femenino de 59 años de edad con antecedente de evento vascular cerebral isquémico hace cinco años, diabetes tipo 2 en con-

Citar como: Ríos-Cruz D, Hernández-Linares FA, Cabrera-Valladares N, Flores-Hidalgo SM, López-Pérez W, Vera-Ruíz MG. Íleo biliar resuelto por laparoscopia. Cir Gen. 2021; 43 (1): 47-50. https://dx.doi.org/10.35366/103914 
trol con hipoglucemiantes orales (metformina de $850 \mathrm{mg}$ cada 12 horas y glibenclamida de 5 mg cada 24 horas), hernia hiatal sin cirugía y cardiopatía isquémica en control con ácido acetilsalicílico de $100 \mathrm{mg}$ vía oral cada 24 horas y atorvastatina de $40 \mathrm{mg}$ vía oral cada 24 horas. Su padecimiento inicia con náuseas y vómito de contenido gastrointestinal color café, que conduce a intolerancia a la vía oral acompañada de dolor en epigastrio referido en intensidad 7/10, automedicada con antiespasmódicos y antiinflamatorios no esteroideos (AINE), con mejoría parcial; 48 horas posteriores al inicio de los síntomas, incrementa la intensidad del dolor y presenta distensión abdominal, así como incapacidad de canalizar gases por recto. A su ingreso al servicio de urgencias, la paciente se encontró deshidratada, con abdomen distendido, doloroso a la palpación superficial y ruidos metálicos. Se colocó sonda nasogástrica cuyo gasto fue fecaloide. Bioquímicamente con falla renal aguda, desequilibrio hidroelectrolítico y acidosis metabólica. Radiográficamente con asas de intestino delgado dilatadas, neumobilia (Figura 1) y datos de oclusión intestinal. Ante esto, se decide exploración quirúrgica laparoscópica, observándose dilatación de asas de intestino delgado hasta un segmento de íleon,

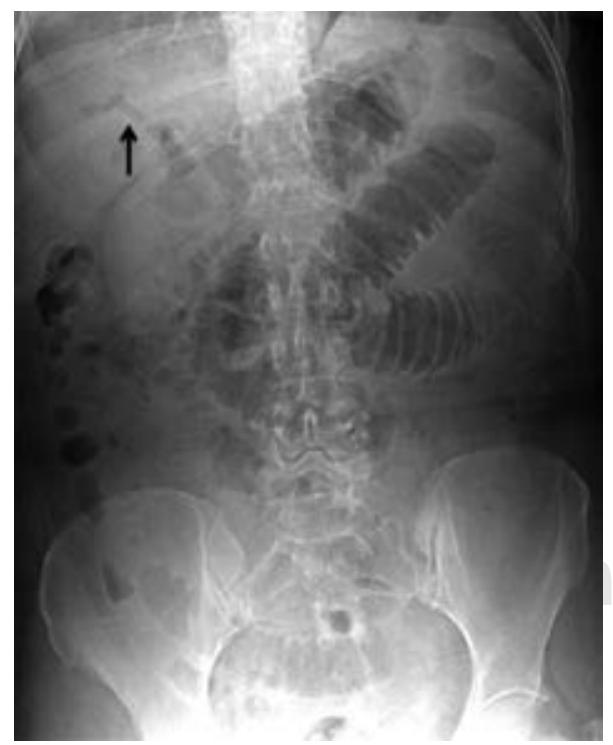

Figura 1: Radiografía simple de abdomen donde se observan las asas de intestino delgado dilatadas. La flecha indica neumobilia.

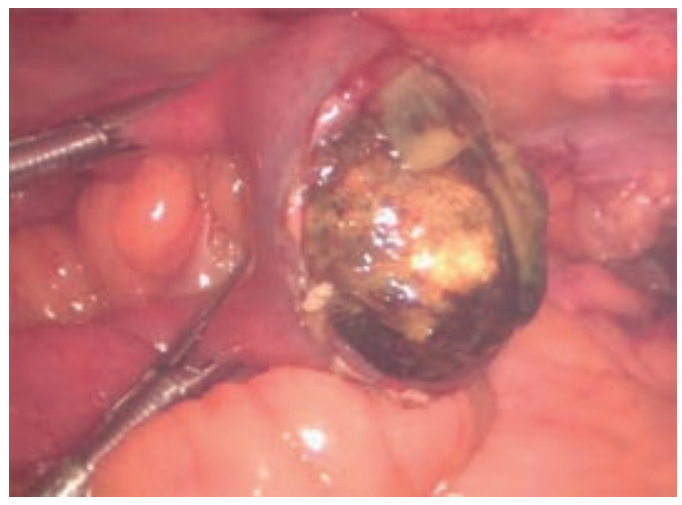

Figura 2: Se observa enterotomía y lito saliendo de la luz del intestino delgado.

localizado a $150 \mathrm{~cm}$ de válvula ileocecal, donde se observa una protuberancia que marca el fin de la dilatación intestinal y que corresponde a un lito de $3.5 \mathrm{~cm}$ enclavado; se realiza enterotomía, extracción de lito y cierre primario en un plano, sutura continua con prolene 2-0 (Figura 2). En el transoperatorio, la paciente se comportó con inestabilidad hemodinámica, por lo que fue necesario iniciar apoyo con norepinefrina (16 mg en $250 \mathrm{ml}$ de solución salina al $0.9 \%$ ) a 8 $\mathrm{ml} / \mathrm{h}$, dosis, que fue en descenso hasta su retiro total en 48 horas. Posteriormente, evolucionó de manera favorable, siendo egresada al quinto día manteniendo estabilidad hemodinámica, tolerando la vía oral, sin datos de respuesta inflamatoria sistémica. Actualmente, a un año de la cirugía, continúa en seguimiento por consulta externa como paciente ambulatoria sin haber presentado complicaciones relacionadas al evento quirúrgico.

\section{DISCUSIÓN}

El íleo biliar representa $0.5 \%$ de las complicaciones de la colelitiasis, es raro y potencialmente grave. Se presenta con mayor frecuencia en mujeres de edad avanzada. Abarca entre 1-4\% del total de las obstrucciones intestinales en el adulto mayor de 65 años, lo que implica un riesgo alto de complicaciones con una mortalidad de 12 a $27 \%$. $^{7}$ Es una patología que no suele ser diagnosticada de forma prematura, debido a la similitud de síntomas que tiene con padecimientos más comunes de abdomen agudo y suele llegarse al diagnóstico por 
hallazgo transoperatorio. Nuestro caso se trata de una mujer de 59 años con comorbilidades asociadas; este dato corresponde con el publicado por Sánchez-Pérez y colaboradores, ${ }^{2}$ quienes estudiaron un grupo de pacientes con diagnóstico de obstrucción intestinal; de los cuales, 10 casos fueron causados por íleo biliar; ocho fueron mujeres y la edad promedio de presentación fue de 61.9 años. Esto hace que los pacientes se presenten al servicio de urgencias en malas condiciones; por lo general, presentan falla renal aguda por deshidratación y desequilibrio ácido-base. En nuestro caso, la paciente se presentó al hospital con un cuadro de 48 horas de evolución sin tolerancia a la vía oral, tiempo en el que no tuvo una ingesta alimentaria adecuada y, por consiguiente, un mal aporte hídrico. Esto condicionó que la paciente llegara a presentar una falla renal aguda por deshidratación y acidosis metabólica junto con datos de respuesta inflamatoria sistémica; hallazgos que suelen tener este tipo de pacientes. ${ }^{2}$

Para que ocurra un íleo biliar, debe existir una fístula bilioentérica, en su mayoría colecistoduodenal (68-95\%), misma que aparece por episodios recurrentes de colecistitis aguda que generan inflamación y adhesiones entre la vesícula biliar y el tracto digestivo. El lito debe tener un diámetro $\geq 2-2.5 \mathrm{~cm}$ para causar obstrucción. ${ }^{8}$ Aproximadamente, sólo 50\% de los pacientes que se presentan con íleo biliar tienen conocimiento de padecer colelitiasis, siendo renuentes a una cirugía electiva. El resto de los pacientes refieren historial de dolor abdominal inespecífico, tratado como dispepsia o trastornos funcionales del colon, y el diagnóstico se obtiene por hallazgo transoperatorio como en este caso; nuestra paciente desconocía el antecedente de colelitiasis y refería dolor abdominal en cuadrantes superiores con la ingesta de alimentos irritantes gástricos, ricos en colecistocinéticos, que se controlaba con antiácidos, inhibidores de la bomba de protones y antiespasmódicos, por lo que nunca buscó atención médica. Este patrón se repetía aproximadamente cada tres semanas durante "toda su vida".

Los hallazgos radiográficos en una proyección simple de abdomen incluyen: neumobilia, datos de obstrucción intestinal, una imagen sugestiva de lito en asas intestinales y cambios en localización de un lito visualizado en una radiografía previa. ${ }^{9-13}$ El uso de la tomografía es un importante apoyo diagnóstico; se ha reportado una sensibilidad, especificidad y precisión diagnóstica de 93, 100 y 99\%, respectivamente. ${ }^{13}$ El tratamiento inicial es la reanimación hídrica, ya que estos pacientes se presentan con deshidratación. El abordaje quirúrgico laparoscópico ofrece más ventajas en comparación con la cirugía abierta, la recuperación requiere menor tiempo. Sin embargo, representa un reto técnico, y más cuando las asas intestinales se encuentran edematosas y dilatadas. ${ }^{14}$ En nuestro caso, preferimos el abordaje laparoscópico sobre el abierto por las ventajas que la cirugía de mínima invasión ofrece. La paciente no presentó complicaciones relacionadas al evento quirúrgico. Existe controversia en cuanto al manejo de la fístula bilioentérica; por un lado, se prefiere únicamente la enterotomía, extracción del lito y cierre primario y, por otro, además de lo anterior, se realiza desmantelamiento de la fístula y colecistectomía. ${ }^{8}$ Se ha reportado que los pacientes de edad avanzada y con múltiples comorbilidades representan un verdadero reto, ya que existe un incremento considerable de fugas, tanto intestinales como biliares, al realizar todos los procedimientos descritos en una sola cirugía. ${ }^{15}$ En nuestro caso, decidimos realizar únicamente enterotomía, extracción del lito y cierre primario debido a las condiciones de la paciente al momento de su ingreso a la sala de quirófano y al comportamiento de inestabilidad hemodinámica transoperatoria. Tomamos la decisión de resolver la urgencia.

De acuerdo con lo reportado por Halabi WJ y colaboradores, $5 \%$ de los pacientes que se sometieron a enterolitotomía como único tratamiento desarrollarán síntomas biliares y 10\% requerirá una operación no planeada. En presencia de cálculos residuales, la prevalencia estimada de recurrencia es de 5 a 17\% y más de la mitad de esas recurrencias serán dentro de los seis meses de la presentación inicial. ${ }^{15}$ Debido a esto, si la vesícula biliar se preserva en la primera cirugía, se debe realizar una colecistectomía diferida.

En su artículo, Salvador Eloy García-Valenzuela y sus colegas presentan un par de casos con íleo biliar resueltos, uno por laparoscopia 
y otro por cirugía abierta convencional. Señalan que hubo escenarios distintos, diferentes factores de comorbilidad y procedimientos quirúrgicos diferentes; pero las pacientes evolucionaron satisfactoriamente y se concluye que ambos procedimientos quirúrgicos son válidos y la elección la hace el cirujano, considerando los factores nutricionales, las comorbilidades de cada paciente y su propia experiencia. ${ }^{16}$ Por lo que tomar la decisión de intervenir por laparoscopia a nuestra paciente estuvo basado en la amplia experiencia en laparoscopia de alta especialidad de todo el equipo, siendo un procedimiento exitoso y demostrando que este padecimiento puede ser resuelto por este método.

\section{CONCLUSIÓN}

El íleo biliar es una patología poco frecuente que afecta principalmente a personas de edad avanzada, predominantemente mujeres. La enterolitotomía con extracción de lito es el procedimiento realizado con mayor frecuencia debido a su baja incidencia de complicaciones.

\section{REFERENCIAS}

1. Martín-Pérez J, Delgado-Plasencia L, Bravo-Gutiérrez A, Burillo-Putze G, Martínez-Riera A, AlarcóHernández $A$, et al. El íleo biliar como causa de abdomen agudo. Importancia del diagnóstico precoz para el tratamiento quirúrgico. Cir Esp. 2013; 91: 485-489.

2. Sánchez-Pérez EA, Álvarez-Álvarez S, Madrigal-Téllez MA, Gutiérrez-Uvalle GE, Ramírez-Velásquez JE, Hurtado-López LM. Gallstone ileus, experience in the Dr. Eduardo Liceaga General Hospital of Mexico. Cir Cir. 2017; 85: 114-120.

3. Zimadlová D, Hoffman P, Bártová J. Gallstone ileus. Case report and review of literature. Folia Gastroenterol Hepatol. 2009; 7: 136-139.

4. Aguilar-Espinosa F, Gálvez-Romero JL, Falfán-Moreno J, Guerrero-Martínez GA, Vargas-Solís F. Sangrado de tubo digestive y delirium, retos en el diagnóstico del íleo biliar: reporte de un caso y revisión de bibliografía. Cir Cir. 2017: 85: 53-57.

5. Kirchmayr W, Muhlmann G, Zitt M, Bodner J, Weiss $\mathrm{H}$, Klaus A. Gallstone ileus: rare and still controversial. ANZ J Surg. 2005; 75: 234-238.
6. Ploneda-Valencia CF, Gallo-Morales M, Rinchon C, Navarro-Muñiz E, Bautista-López CA, De la CerdaTrujillo LF, et al. El íleo biliar: una revisión de la literatura médica. Rev Gastroenterol Mex. 2017; 82: 248-254.

7. García-Marín A, Pérez-López M, Pérez-Bru S, Compañ-Rosique A. Gallstone ileus, an uncommon cause of bowel obstruction. Rev Gastroenterol Mex. 2014; 79: 211-213.

8. Dai XZ, Li GQ, Zhang F, Wang XH, Zhang CY. Gallstone ileus: case report and literature review. World J Gastroenterol. 2013; 19: 5586-5589.

9. Al-Obaid O. Gallstone ileus: a forgotten rare cause of intestinal obstruction. Saudi J Gastroenterol. 2007; 13: 39-42.

10. Ripollés T, Miguel-Dasit A, Errando J, Morote V, GómezAbril SA, Richart J. Gallstone ileus: increased diagnostic sensitivity by combining plain film and ultrasound. Abdom Imaging. 2001; 26: 401-405.

11. Clavien PA, Richon J, Burgan S, Rohner A. Gallstone ileus. Br J Surg. 1990; 77: 737-742.

12. Lassandro F, Gagliardi N, Scuderi M, Pinto A, Gatta G, Mazzeo R. Gallstone ileus analysis of radiological findings in 27 patients. Eur J Radiol. 2004; 50: 23 29.

13. Yu CY, Lin CC, Shyu RY, Hsieh CB, Wu HS, Tyan YS, et al. Value of CT in the diagnosis and management of gallstone ileus. World J Gastroenterol. 2005; 11: 2142-2147.

14. Sarli L, Pietra N, Costi R, Gobbi S. Gallstone ileus: laparoscopic-assisted enterolithotomy. J Am Coll Surg. 1998; 186: 370-371.

15. Halabi WJ, Kang CY, Ketana N, Lafaro KJ, Nguyen VQ, Stamos MJ, et al. Surgery for gallstone ileus: a nationwide comparison of trends and outcomes. Ann Surg. 2014; 259: 329-335.

16. García-Valenzuela SE, Trujillo-Bracamontes FS, Quintero-García B, Ríos-Beltrán JC, Valdez-Avilés D. Obstrucción intestinal secundaria a íleo biliar: reporte de dos casos. Rev Esp Med Quir. 2015; 20: 111-115.

Consideraciones y responsabilidad ética: Este artículo cuenta con la autorización del paciente y se siguieron las regulaciones de investigación bioética.

Conflicto de intereses: Los autores declaran que no existe ningún conflicto de intereses en la realización del trabajo.

Correspondencia:

Daniel Ríos-Cruz

Hospital Center Vista Hermosa.

Consultorio 109.

E-mail:dr_rioscruz@outlook.com 